02

\title{
Генерация капельных квазибесселевых пучков при помощи полупроводникового лазера
}

\author{
(C) Д.В. Чистяков ${ }^{1}$, С.Н. Лосев ${ }^{2}$, С.Х. Абдулразак ${ }^{2}$, В.Ю. Мыльников ${ }^{2}$, Е.А. Когновицкая ${ }^{3}$, Ю.М. Задиранов ${ }^{2}$, \\ Н.Г. Дерягин ${ }^{2}$, В.В. Дюделев ${ }^{2}$, В.И. Кучинский ${ }^{2}$, Г.С. Соколовский ${ }^{2}$ \\ ${ }^{1}$ Университет ИТМО, \\ 197101 Санкт-Петербург, Россия \\ ${ }^{2}$ ФТИ им. А.Ф. Иофрфе, \\ 194021 Санкт-Петербург, Россия \\ ${ }^{3}$ Санкт-Петербургский государственный электротехнический университет „ЛЭТИ“, \\ 197022 Санкт-Петербург, Россия \\ e-mail: gs@mail.ioffe.ru
}

Поступила в редакцию 05.07.2019 г.

В окончательной редакции 15.07.2019 г.

Принята к публикации 23.07.2019 г.

\begin{abstract}
Продемонстрирован способ генерации капельных квазибесселевых пучков при помощи конической линзы с существенно скругленной вершиной. Исследование продольного распределения интенсивности полученного квазибесселева пучка показало, что из-за интерференции двух волновых фронтов, возникающих при прохождении образующего пучка через скругленный аксикон, в результирующем пучке появляются периодические пульсации интенсивности, визуально напоминающие „капли“ света. Такие световые лучи могут быть использованы для микроманипуляций биологическими объектами и в сверхразрешающей микроскопии. При этом использование аксикона со скругленной вершиной для формирования капельного пучка позволяет значительно упростить и миниатюризировать экспериментальную установку, что открывает путь для многих практических применений.
\end{abstract}

Ключевые слова: полупроводниковый лазер, бесселевы пучки, капельные пучки, аксикон.

DOI: $10.21883 /$ OS.2019.11.48515.229-19

В настоящее время полупроводниковые лазеры активно вытесняют газовые и твердотельные благодаря компактным размерам, невысокой стоимости и высокой эффективности. Однако применение полупроводниковых лазеров в различных областях науки и техники ограничено из-за низкой плотности мощности излучения. Основным недостатком мощных полупроводниковых лазеров является низкое пространственное качество излучения, связанное с многомодовостью выходного пучка, не позволяющее получить высокую плотность мощности в сфокусированном пучке.

Для описания качества лазерного излучения используется параметр распространения $M^{2}$ [1]. Этот параметр описывает численное увеличение размера фокусного пятна в $M^{2}$ раз по отношению к размеру пятна при фокусировке идеального гауссова луча той же длины волны, той же оптической системой. Для мощных полупроводниковых лазеров типичное значение $M^{2}$ находится в пределах нескольких десятков, что снижает достижимую плотность мощности на два-три порядка.

Решить проблему низкой плотности мощности сфокусированного излучения полупроводникового лазера позволяет „интерференционная“ фокусировка [2-4] за счет генерации бесселевых пучков $[5,6]$. В сечении бесселев пучок представляет собой яркое пятно, окруженное кольцами, интенсивность которых описывается функцией Бесселя первого рода нулевого порядка. По- перечный размер такого пятна может быть значительно меньше, чем при традиционной фокусировке [4,7]. Также бесселев пучок имеет свойство самовосстанавливаться после встречи с препятствием [8]. Дистанция восстановления зависит от размера препятствия и угла при вершине конической линзы (аксикона). Благодаря описанным выше свойствам бесселев пучок особенно привлекателен для бесконтактных манипуляций различными микроскопическими объектами $[9,10]$, при этом одним бесселевым пучком можно манипулировать сразу несколькими частицами [11].

Здесь следует пояснить термины „нерасходящиеся пучки“ и „бесселевы пучки“, применяемые в настоящей работе. Общеизвестно, что любой пучок с конечной мощностью излучения испытывает в дальней зоне дифракционное расплывание, а также, что „идеальный бесселев пучок обладает бесконечно большой мощностью и потому представляет физически нереализуемую абстракцию подобно плоской волне [12]. Основная часть мощности „идеального“ бесселева пучка содержится в его бесконечно далеких крыльях, обрезание которых качественно меняет поведение пучка в дальней зоне. Поэтому его „нерасходящийся“ центральный луч имеет конечную длину распространения, определяемую апертурой и углом при вершине аксикона. На эти очевидные обстоятельства обращалось внимание во многих публикациях, включая самые ранние, посвященные данной 
теме $[5,12]$. В связи с этим в настоящей работе мы будем в основном пользоваться термином „квазибесселев пучок

Помимо изучения классических квазибесселевых пучков, имеющих протяженный нерасходящийся центральный луч, появились публикации, авторами которых исследуются квазибесселевы пучки, центральный луч которых распространяется прерывисто в виде „световых капель“. В работе [13] продемонстрирована генерация „капельной“ структуры луча при помощи интерференции двух сонаправленных квазибесселевых пучков. Также экспериментально получены нерасходящийся трехмерный монохроматический „капельный“ квазибесселев луч [14] и „капельный“ луч с подавлением боковых колец [15]. Однако стоит отметить, что генерация „капельных“ квазибесселевых лучей в рассмотренных публикациях реализована при помощи громоздкой и дорогой оптической системы на основе пространственного светового модулятора (SLM, spatial light modulator).

В настоящей работе развивается предложенный ранее [16] метод генерации квазибесселевых пучков с „капельной“ структурой центрального луча при помощи конической линзы со скругленной вершиной. Таким образом можно значительно упростить и миниатюризировать экспериментальную установку, что открывает путь для многих практических применений.

Схематическое изображение экспериментальной установки приведено на рис. 1. Излучение полупроводникового лазера с волоконным выводом с длиной волны $\lambda=1064 \mathrm{~nm}$ коллимируется объективом $20 \times$ и, проходя через коническую линзу (аксикон) с углом при вершине $\alpha=160^{\circ}$, формирует квазибесселев пучок. Система наблюдения состоит из фокусирующего объектива $20 \times$ и фотокамеры, которые закреплены на микропозиционере, обеспечивающем продольное перемещение системы.

Исследование полученного пучка проходит следующим образом: производится фотосъемка поперечного распределения интенсивности через равные интервалы в $10 \mu \mathrm{m}$. Полученный набор снимков при помощи программы ImageJ [17] преобразуется в продольное распределение интенсивности исследуемого пучка (рис. 2,a) и строится график зависимости интенсивности пучка от дистанции распространения (рис. $2, b$ ).

Исследование продольного распределения интенсивности генерируемого квазибесселева пучка показало, что центральное яркое пятно пучка распространяется прерывисто в виде „световых капель“. Появление „капельной“ структуры (осцилляции интенсивности излучения) квазибесселева пучка связано с тем, что из-за скругления вершины конической линзы наблюдается интерференция плоского волнового фронта квазибесселева пучка и сферического фронта гауссова пучка. Бесселев пучок генерируется конической частью аксикона, а гауссов пучок формируется его сферической частью, расположенной в области вершины. Поэтому управление размером скругленной области вершины аксикона, радиусом ее скругления, а также углом аксикона позволяет изменять характеристики „капельной“ структуры квазибесселева пучка.

Влияние этих параметров на продольное распределение интенсивности „капельного“ пучка изучалось экспериментально путем исследования влияния расходимости образующего луча, которая варьировалась за счет изменения расстояния между источником излучения и конической линзой. Схема экспериментальной установки представлена на рис. 1 (коллимирующая линза не учитывается). При помощи микропозиционера аксикон перемещается от лазера на произвольные расстояния (7, 12, 17 и $32 \mathrm{~mm})$. При этом на расстоянии $32 \mathrm{~mm}$ от лазера аксикон полностью заливается лазерным излучением. Далее на каждом из расстояний аксикона от лазера производится фотосъемка поперечного распределения интенсивности через равные интервалы в $50 \mu \mathrm{m}$. Полученные наборы снимков при помощи программы ImageJ [17] преобразуются в продольные распределения интенсивности исследуемого пучка и строятся графики зависимостей интенсивности пучка от дистанции распространения (рис. 3).

Для численного моделирования „капельного“ пучка необходимо использовать математическую модель, в которой аксикон со скругленной вершиной представляется в виде гиперболоида вращения, подобно описанному в работе [18]. Предположим, что на поверхность аксикона падает расходящийся гауссов пучок, амплитуда напряженности электрического поля которого будет иметь вид [19]

$$
E_{0}(r)=A \exp \left(-\frac{r^{2}}{w^{2}}+\frac{i k r^{2}}{2 R}\right),
$$

где $A-$ произвольная комплексная константа, $r-$ модуль радиус-вектора в поперечной плоскости, $w-$ ширина гауссова пучка в плоскости аксикона, $k=2 \pi / \lambda-$ волновой вектор света в вакууме, $R$ - радиус кривизны волнового фронта пучка. Действие аксикона учитывается за счет умножения распределения (1) на фазовую маску $F(r)$ вида

$$
F(r)=\exp (-i k(n-1) f(r)),
$$

где $n$ - показатель преломления аксикона, а $f(r)$ кривая, задающая форму поверхности аксикона, которая в случае идеально острой вершины дается выражением

$$
f(r)=\frac{r}{\operatorname{tg}(\alpha / 2)}
$$

где $n-$ угол при вершине аксикона.

Из-за скругления вершины вблизи центра $f(r) \approx r^{2}$, что соответствует фазовой маске тонкой линзы, при этом формула (3) становится применима только вдалеке от скругления. Чтобы сшить область вблизи скругления аксикона с областью вдалеке от нее, использована аппроксимация кривой поверхности $f(r)$ гиперболой, 


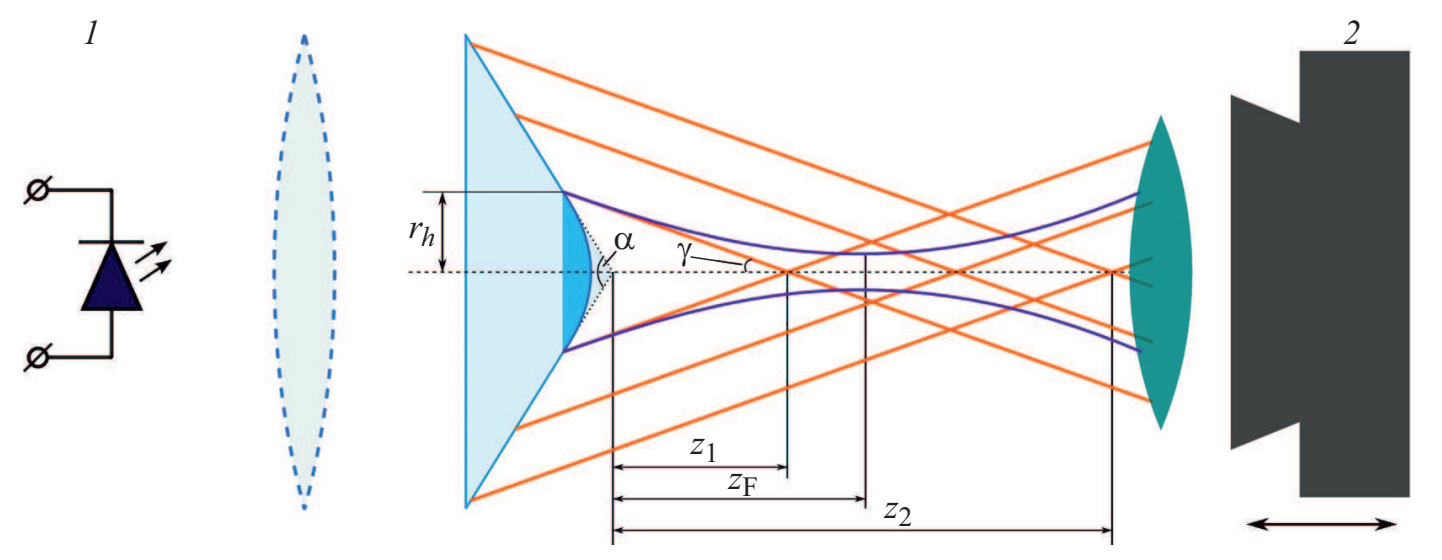

Рис. 1. Схема экспериментальной установки: 1 - полупроводниковый лазер с волоконным выводом излучения, $2-$ цифровая камера, $r_{h}$ - радиус скругленной области аксикона, $z(1(2))$ - расстояние от вершины аксикона до начала (конца) геометрической области формирования квазибесселева пучка, $z_{\mathrm{F}}-$ расстояние от вершины аксикона до фокусного пятна, $\alpha-$ угол при вершине аксикона.
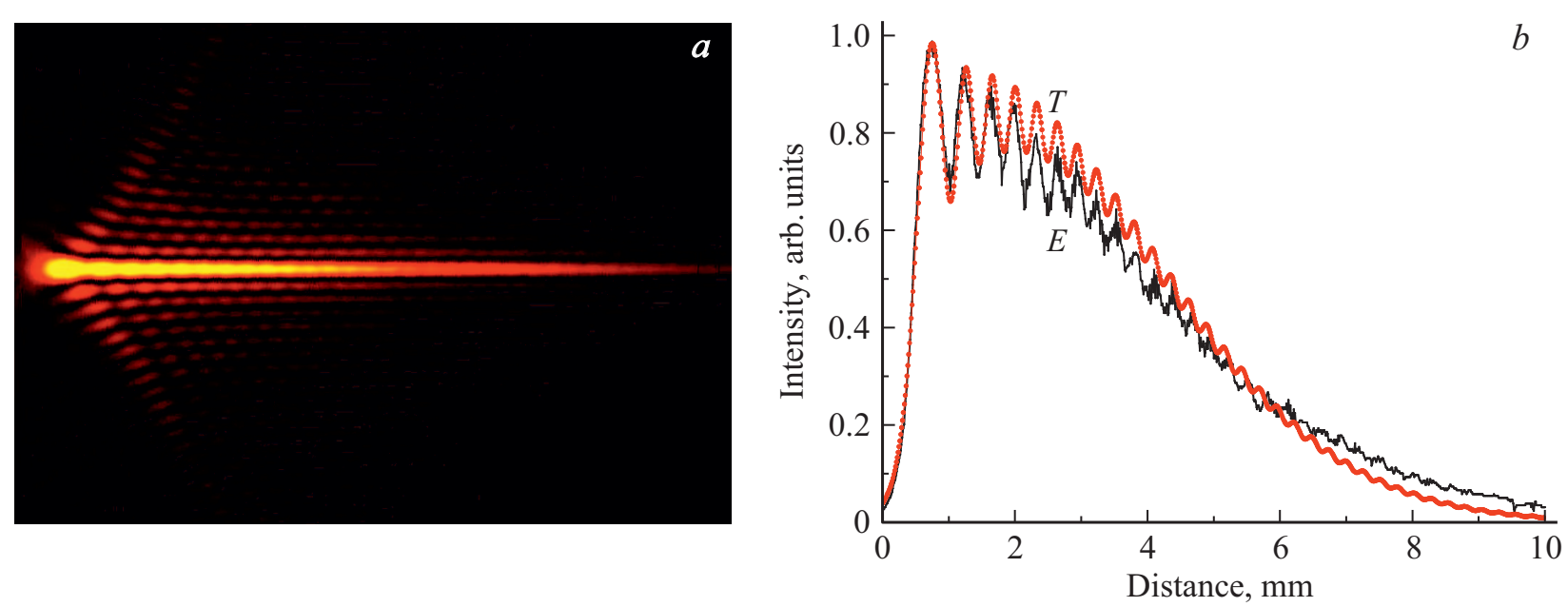

Рис. 2. (a) Продольное распределение интенсивности исследуемого пучка; $(b)$ график зависимости интенсивности пучка от дистанции распространения: $E$ - эксперимент, $T$ - теоретический расчет.

подобно описанной в работе [18]:

$$
f(r)=\frac{\sqrt{r^{2}+r_{h}^{2}}}{\operatorname{tg}(\alpha / 2)},
$$

где параметр $r_{h}$ соответствует радиусу скругленной области. Дальнейшее свободное распространение излучения за выходной гранью аксикона определяется дифракционным интегралом Френеля [19]. Таким образом, выражение, определяющее распределение поля на оси $U(z)$, в параксиальном случае будет иметь вид

$$
\begin{aligned}
U(z)= & \frac{A k}{z} \int_{0}^{\infty} \exp \left[-\left(\frac{1}{w^{2}}-\frac{i k}{2}\left(\frac{1}{R}+\frac{1}{z}\right)\right) r^{2}\right. \\
& \left.-i k \gamma \sqrt{r^{2}+r_{h}^{2}}\right] r d r,
\end{aligned}
$$

где введен угол геометрического схождения пучков после аксикона:

$$
\gamma=\frac{n-1}{\operatorname{tg}(\alpha / 2)}
$$

На рис. 2 представлен результат численного моделирования для коллимированного входящего пучка, полученный по формуле (5). Видно, что результат моделирования хорошо согласуется с экспериментальной кривой. Для теоретического расчета использовались следующие параметры: ширина пучка $w=620 \mu \mathrm{m}$, радиус кривизны волнового фронта $R$ стремится к бесконечности, длина волны света $\lambda=1064 \mathrm{~nm}, \gamma=0.084, r_{h}=175 \mu \mathrm{m}$.

На рис. 3 представлено осевое распределение интенсивности, полученное в эксперименте и при помощи численного моделирования, для конечного значения радиуса кривизны волнового фронта $R \approx L$, где $L-$ расстояние от торца волоконного вывода лазера до аксикона, равное 

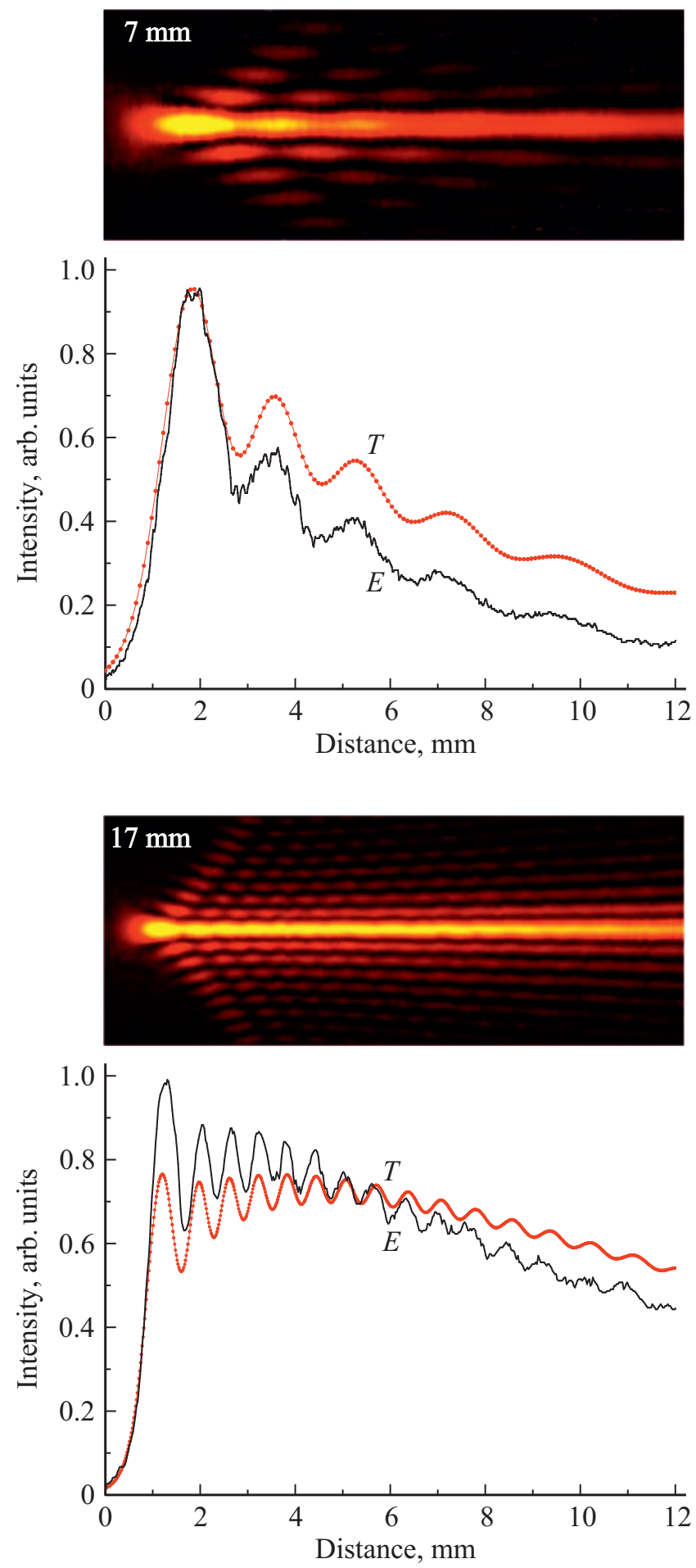
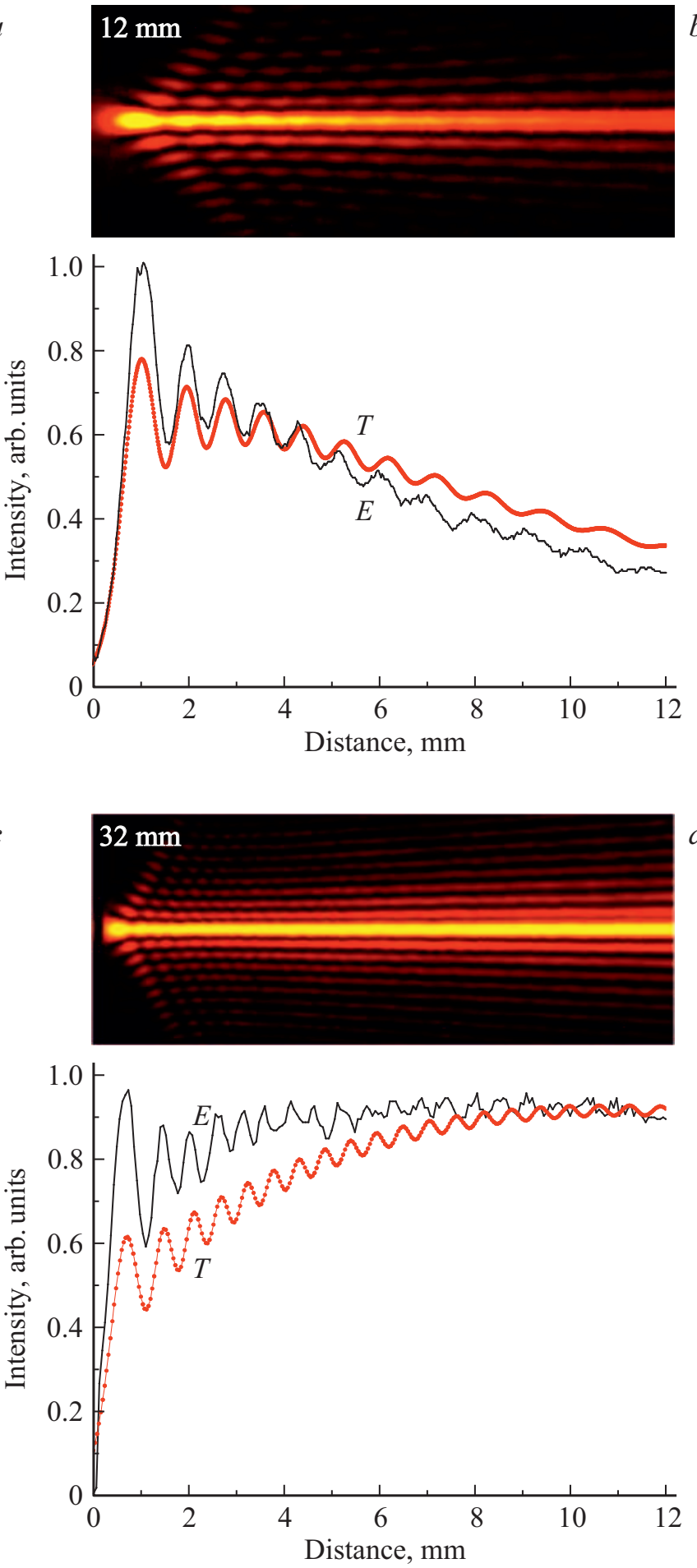

Рис. 3. Продольные распределения интенсивностей исследуемого пучка и графики зависимостей интенсивности пучка от дистанции распространения для $7(a), 12(b), 17(c)$ и $32 \mathrm{~mm}(d) . E$ - эксперимент, $T$ - теоретический расчет.

7, 12, 17 и $32 \mathrm{~mm}$. Ширина пучка на аксиконе определяется расходимостью и составляет $\omega=0.7,1.16,1.6$ и $3 \mathrm{~mm}$ для каждого из расстояний соответственно. Теоретические и экспериментальные зависимости демонстрируют очевидное качественное согласие, выражающееся, в частности, в совпадении периода осцилляций для теоретического расчета и экспериментальной кривой. Некоторое количественное рассогласование скорости спада интенсивности центрального пятна квазибесселева пучка может быть объяснено отличием экспериментального распределения интенсивности образующего луча от гауссова, а также отличием реальной формы скруг- 
ленной поверхности аксикона $f(r)$ от выбранной ранее аппроксимации гиперболоидом вращения [18].

Исследованные в работе квазибесселевы пучки с „капельной“ структурой центрального пятна могут быть эффективно использованы для оптического захвата и манипулирования микроскопическими объектами. При этом для объектов с высоким показателем преломления может использоваться область, где интенсивность центрального пятна квазибесселева пучка максимальна [20]. Объекты с показателем преломления ниже показателя преломления среды могут удерживаться за счет зажигания боковых колец пучка, в то время как интенсивность центрального пятна минимальна. Использование двух встречных квазибесселевых пучков позволяет создать „бутылочный“ луч без использования пространственного светового модулятора, подобно описанному в работе [13].

При наблюдении и регистрации микроскопических изображений биологических объектов в основном используются оптические системы, предельное увеличение которых зависит от дифракции, поглощения и рассеянии сфокусированного светового потока на слоях толстых непрозрачных объектов и объектов со сложной внутренней структурой. Дифракция вынуждает использовать громоздкую оптику и объективы с большой апертурой для получения высокого разрешения. Поглощение и рассеяние, в свою очередь, ограничивают проникновение сфокусированного луча в слои образца.

Стандартные методы наблюдении и регистрации увеличенных изображений объектов могут проникать на глубину не более сотни микрометров внутрь биологического образца [21]. Нелинейные оптические эффекты могут увеличивать глубину фокусировки системы отображения, однако это требует использования специальных материалов [22].

Проблемы, связанные с дифракцией, поглощением и рассеянием сфокусированного светового потока на исследуемых слоях объектов, могут быть эффективно решены за счет использования нерасходящегося трехмерного монохроматического „капельного“ квазибесселева луча [14]. Квазибесселев луч способен проникать глубоко в исследуемый образец, что позволяет получить изображение без искажений даже для толстых непрозрачных объектов. При этом разрешение „капельного“ пучка сравнимо с разрешением оптической системы на основе гауссова пучка. „Световые капли“ сохраняют свой пространственный профиль и способны создать трехмерное освещение внутри полых объектов [14]. Нерасходимость капельного пучка является ключевым фактором для трехмерной флуоресцентной [23] и оптической микроскопии [24], так как обеспечивает высокий контраст изображения и баланс освещения исследуемого объекта, а также минимизирует шумовой фон. Также капельные пучки могут быть использованы в оптогенетике для изучения динамики движения живых клеток [25] и нейронной активности [26].
Таким образом, исследованные в работе „капельные“ квазибесселевы пучки, полученные при помощи конической линзы со скругленной вершиной, могут быть эффективно использованы для микроманипуляций биологическими объектами и в сверхразрешающей микроскопии. Изучение распределения интенсивности полученного луча показало, что скругление вершины конической линзы приводит к возникновению в результирующем световом луче периодических пульсаций интенсивности, визуально напоминающих „капли“ света. При этом использование конической линзы со скругленной вершиной позволяет значительно упростить и миниатюризировать экспериментальную установку по сравнению с применением громоздкой и дорогой оптической схемой на основе пространственного светового модулятора.

\section{Конфликт интересов}

Авторы заявляют, что у них нет конфликта интересов.

\section{Список литературы}

[1] Lasers and laser-related equipment - Test methods for laser beam widths, divergence angles and beam propagation ratios. ISO 11146-2. BSI, 2015. $26 \mathrm{p}$.

[2] Sokolovskii G.S., Melissinaki V., Fedorova K. A., Dudelev V.V., Losev S.N., Bougrov V.E., Sibbett W., Farsari M., Rafailov E.U. // Sci. Rep. 2018. V. 8. P. 14618.

[3] Sokolovskii G.S., Butkus M., Losev S.N., Dudelev V.V., Deryagin A.G., Kuchinskii V.I., Sibbett W., Rafailov E.U. // Proc. SPIE. 2012. V. 8242. P. 82420T (1.7).

[4] Sokolovskii G.S., Dudelev V.V., Losev S.N., Deryagin A.G. Kuchinskii V.I., Sibbett W., Rafailov E.U. // Tech. Phys. Lett. 2012. V. 38. N 5. P. 402-404.

[5] Durnin J. // J. Opt. Soc. Am. A. 1987. V. 4. N 4. P. 651-654.

[6] Zel'dovich B.Ya., Pilipetskii T.A. // Izvestia Vyzov. Radiophysics. 1966. V. 9. N 1. P. 95-101.

[7] Соколовский Г.С., Дюделев В.В., Лосев С.Н., Дерягин А.Г., Винокуров Д.А., Лютеикий А.В., Пихтин Н.А., Слипченко С.О., Тарасов И.С., Золотовская С.А., Рафаилов Э.У., Кучинский В.И., Сиббет В. // Письма в ЖТФ. 2010. Т. 36. B. 1. C. 22-30; Sokolovskii G.S., Dudelev V.V., Losev S.N., Deryagin A.G., Vinokurov D.A., Lyutetskii A.V., Pikhtin N.A., Slipchenko S.O., Tarasov I.S., Zolotovskaya S., Rafailov E.U., Kuchinskii V.I., Sibbett W. // Tech. Phys. Lett. 2010. V. 36. N 1. P. 9-12.

[8] Bouchal Z., Wagner J., Chlup M. // Opt. Commun. 1998. V. 151. P. 207-211.

[9] Garcés-Chávez V., Mc Gloin D., Melville H., Sibbett W., Dholakia K. // Nature. 2002. V. 419. N 6903. P. 145-147.

[10] Arlt J., Graces-Chavez V., Sibbett W., Dholakia K. // Opt. Commun. 2001. V. 197. P. 239-254.

[11] MacDonald M.P., Spalding G.C., Dholakia K. // Nature. 2003. V. 426. N 6965. P. 421-424.

[12] Ananyev Yu.A. // Opt. Spectr. 1988. V. 64. N 6. P. 1211.

[13] Li L., Lee W.M., Xie X., Krolikowski W., Rode A.V., Zhou J. // Opt. Lett. 2014. V. 39. N 8. P. 2278-2281.

[14] Antonacci G., Di Domenico G., Silvestri S., DelRe E., Ruocco G. // Sci. Rep. 2017. V. 7(1). P. 17. 
[15] Di Domenico G., Ruocco G., Colosi C., DelRe E., Antonacci G. // Sci. Rep. 2018. V. 8. P. 17178.

[16] Losev S.N., Abdulrazak S.H., Chistyakov D.V., Myl'nikov V.Y., Kognovitskaya E.A., Berkutov I.V., Zadiranov Y.M., Deryagin N.G., Dudelev V.V., Kuchinskii V.I., Sokolovskii G.S. // Tech. Phys. Lett. 2018. V. 44. N 10. P. 887-889.

[17] Image J. [Electronic resource]. URL: https://imagej.nih.gov/ij/X

[18] Brzobohatý O., Cižmźr T., Zemánek P. // Opt. Express. 2008. V. 16. N 17. P. $12688-12700$.

[19] Born M., Wolf E. Principles of Optics. Cambridge Univ. Press, 1999.

[20] Sokolovskii G.S., Dudelev V.V., Losev S.N., Soboleva K.K., Deryagin N.G., Kuchinskii V.I., Sibbett W., Rafailov E.U. // J. Phys. Conf. Ser. 2014. V. 572. P. 012039.

[21] Keller P.J. // Science. 2013. V. 340. P. 6137.

[22] DelRe E., Spinozzi E., Agranat A.J., Conti C. // Nat. Photon. 2011. V. 5. P. 39.

[23] Purnapatra S.B., Bera S., Mondal P.P. // Sci. Rep. 2012. V. 2. P. 692.

[24] Levoy M., Ng R., Adams Footer M., Horowitz M. // ACM Trans. Graph. 2006. V. 25. P. 3.

[25] Planchon T.A. et al. // Nature Methods. 2011. V. 8. P. 417-423.

[26] Prevedel R. et al. // Nature Methods. 2014. V. 11. P. 727-730. 